\title{
Passive Opto-Antenna using Air-Filled Substrate-Integrated-Waveguide Technology
}

\author{
Olivier Caytan*, Laurens Bogaert*†, Haolin Li*, Joris Van Kerrebrouck*, Sam Lemey*, \\ Johan Bauwelinck*, Piet Demeester*, Guy Torfs*, Dries Vande Ginste*, and Hendrik Rogier* \\ ${ }^{*}$ IDLab, Department of Information Technology (Email: Olivier.Caytan@UGent.be) \\ ${ }^{\dagger}$ Photonics Research Group, Department of Information Technology \\ Ghent University/imec, 9000 Ghent, Belgium
}

\begin{abstract}
In this contribution, we propose a novel extensive full-wave/circuit cosimulation model to analyze the performance of an opto-electronic fully passive downlink remote antenna unit. The vertical-cavity surface-emitting laser, the optical back-toback connection, the photodetector, the lumped/distributed matching network, and the air-filled substrate-integrated-waveguide antenna are described in a single, complete model, which also incorporates the spurious radiation by the distributed matching network and any remaining impedance mismatch of the antenna.
\end{abstract}

\section{INTRODUCTION}

Several next-generation wireless communication systems, including [1], will benefit from radio over fiber (RoF) [2] technologies. Optical fibers allow transportation of highbandwidth radio-frequency (RF) signals over great distances with minimal propagation loss, while being immune against electromagnetic interference. Furthermore, several components, such as the RF transceivers, can be centralized at a central office, which considerably reduces the complexity of the remote antenna units (RAUs) and facilitates high cell densification. Within a radio-frequency over fiber (RFoF) scheme, the RF signals are immediately modulated on the optical carrier, such that RAUs with minimal complexity are obtained, which only need to perform conversion between the optical and electrical domain.

In [1], a highly specialized wireless communication system is proposed, targeting a futuristic factory, roamed through by many autonomous robots. To provide each robot with a highbandwidth and low-latency wireless access, the floor is equipped with a huge matrix of RAUs, with each individual RAU serving a small attocell. Such a solution is only technically and economically feasible when low-cost and low-power RAUs are available. To this end, [3] proposes a fully passive downlink remote antenna unit (DL-RAU), driven by a single optical fiber and tailored towards [1]. Fully passive and robust operation is obtained by employing a zero-biased photodetector and by omitting electrical amplification. In contrast to previous works [4]-[7], the power radiated by the unit is optimized in a specified frequency bandwidth by means of a nearly lossless lumped/distributed matching network, which transforms the antenna's input impedance $(50 \Omega)$ to match the output impedance of the photodetector.

To further enhance the radiated power of the unit, a highly efficient cavity-backed slot antenna based on airfilled substrate-integrated-waveguide (AFSIW) technology [8] is adopted. This technology allows to realize low-loss waveguide structures by means of standard printed circuit board (PCB) laminates, similar to conventional rectangular metallic waveguides and employs air as the medium for the fields. Several AFSIW components have been proposed, including transitions to microstrip, filters [9] and antennas [8]. AFSIW implementations combine the benefits of conventional waveguide technology, most importantly high shielding and low propagation loss, with those of low-profile and cost-effective planar implementations.

In [3], the performance of the proposed DL-RAU was estimated by connecting separate models of the AFSIW antenna and the optoelectronic conversion circuit, such that, among other things, the influence of the spurious radiation by the distributed matching network was not taken into account. Therefore, this contribution extends [3] with results of a more extensive full-wave/circuit cosimulation model of the DLRAU. Section II summarizes the design process of the DLRAU and presents the improved simulation model. Section III formulates a conclusion.

\section{Design of the Passive DL-RAU}

\section{A. High-level Overview}

A high-level functional overview of the DL-RAU [3] is presented in Fig. 1a. A $0.85 \mu \mathrm{m}$ vertical-cavity surface-emitting laser (VCSEL), multi-mode fiber (MMF) and photodetector constitute an intensity modulation and direct detection link. The VCSEL is biased by a current $I_{B}$ and directly modulated by an RF signal, intended for wireless transmission, within the small-signal regime. The generated optical signal is launched into the MMF and illuminates a zero-biased photodetector. The fiber is assumed to remain sufficiently short such that propagation loss and dispersion is negligible. The resulting photocurrent is proportional to the received optical power (responsivity $R$ ) and consists of a DC component $I_{D C}$, arising from the average optical power, and an RF component $I_{R F}$, reconstructing the RF signal. To radiate as much RF power as possible in the absence of an electrical amplifier, a nearly lossless lumped/distributed two-port network ensures impedance matching between the photodetector and the $50 \Omega$ antenna within the RF signal frequency band $(3.3 \mathrm{GHz}$ to $3.7 \mathrm{GHz}$ ). When small-signal operation of the VCSEL and photodetector is ensured, the RF power radiated by the unit is 
increased by increasing the RF power of the signal modulating the VCSEL. Ultimately, the transmit power and useful range of the DL-RAU are limited by nonlinear distortion introduced by the VCSEL and the photodetector.

\section{B. AFSIW Cavity-Backed Slot Antenna as Platform}

The DL-RAU [3], presented schematically in Fig. 2, consists of a stack of four dual-layer PCBs and is based on the AFSIW cavity-backed slot antenna proposed in [8]. Alignment, fixation and electrical contact of the PCBs is obtained by means of M3 screws routed through non-plated holes, present on each PCB. The two identical, central $1.55 \mathrm{~mm}$ FR-4 cavity PCBs constitute the rectangular air-filled cavity of dimensions $90.0 \mathrm{~mm} \times 47.4 \mathrm{~mm}$. The cavity's inner edges are metalized, such that the lossy FR-4 substrate is not penetrated by the fields of the AFSIW antenna. The cavity PCBs are covered by the ground plane of the feed PCB on one side, and the copper slot layer of the slot PCB on the other side. The slot and feed PCBs are integrated on $508 \mu \mathrm{m}$ Rogers 4350B high-frequency laminates. All copper layers have a thickness of $35 \mu \mathrm{m}$.

The photodetector and matching network, collectively referred to as the optoelectronic conversion circuit (OECC), are integrated on the feed PCB, exploiting the AFSIW cavitybacked slot antenna as a platform. The OECC is described in Subsection II-C. The OECC excites the AFSIW cavity by means of a feed probe soldered to an annular ring with a diameter of $4.20 \mathrm{~mm}$ on the ring layer of the slot PCB. As shown in the cutaway detail in Fig. 2, a clearance hole with a diameter of $2.30 \mathrm{~mm}$ is preserved, such that the probe does not make direct contact with the slot layer and the AFSIW cavity is capacitively fed, similar to [10]. The overlap between the annular ring and slot layer forms a capacitor, indicated by $C_{F}$ in Fig. 2, which conducts the RF currents applied by the probe, exciting the air-filled cavity. In contrast, the DC component of the photocurrent is blocked, such that it can be accurately measured by means of a dedicated bias tee.

A rectangular aperture $(88.0 \mathrm{~mm} \times 8.2 \mathrm{~mm})$ in the slot layer allows the AFSIW cavity to radiate into free-space. Due to its length, this radiating slot divides the rectangular AFSIW cavity into two coupled half-mode AFSIW cavity resonators [8], which resonate at slightly different frequencies, greatly enhancing the antenna's impedance bandwidth. Also owing to its thick air substrate, the AFSIW antenna achieves an ultra-wide impedance bandwidth $(10 \mathrm{~dB}$ return loss w.r.t. $50 \Omega$ ) ranging from $3.1 \mathrm{GHz}$ to $3.9 \mathrm{GHz}$, after optimization with the frequency solver of CST Microwave Studio 2017. Details on the optimization are provided in [3]. The RF signal frequency band is covered with adequate margins, accounting for fabrication errors. Although a multi-moding technique is applied to extend the antenna's bandwidth, a stable broadside gain of $8.0 \mathrm{dBi}$ is predicted in the entire frequency band.

\section{Optoelectronic Conversion Circuit}

The OECC contains a lumped/distributed matching network, which transforms the $50 \Omega$ input impedance of the antenna to match the photodetector's output impedance. This circuit is implemented on the feed PCB and is shown in detail in
Fig. 3. In contrast to [3], the performance of the DL-RAU is assessed with a complete full-wave/circuit cosimulation model (presented schematically in Fig. 1b), encompassing both the AFSIW antenna and the OECC. Such a complete model provides a more accurate description of the DL-RAU, as it takes the spurious radiation by the distributed matching network into account, as well as the slight frequency-dependent antenna impedance, which is not exactly $50 \Omega$ in the entire band. The components in blue, which include the small-signal equivalent circuit models of the VCSEL and photodetector, the optical back-to-back connection, and the models for the $7.2 \mathrm{nH}$ and $34.0 \mathrm{nH}$ chip inductors, are taken into account in the circuit simulation. The components in black constitute the distributed part of the matching network, and are included in the full-wave simulation, in addition to the $50 \Omega$ AFSIW antenna.

The small-signal output impedance of the employed 25 Gbps PIN photodiode is measured by means of a calibrated microwave probe, indicating that the component at $0 \mathrm{~V}$ bias is accurately modeled by a junction capacitance of $135.6 \mathrm{fF}$ in series with a resistance of $22.1 \Omega$. Based on this model, a lumped/distributed implementation of a third order bandpass $(3.3 \mathrm{GHz}-3.7 \mathrm{GHz})$ Chebyshev impedance matching network [11] is devised. This lossless network maximizes the minimum transducer gain within the considered frequency band, for the photodetector model serving as the generator and the $50 \Omega$ antenna impedance as the load [11]. As shown in Fig. 1b, the lumped/distributed implementation consists of a $7.2 \mathrm{nH}$ chip inductor, two microstrip quarter-wavelength transformers (QWLTs), two open shunt radial stubs with very low characteristic impedance, and an electrically short grounded coplanar waveguide (GCPW) stub. By shorting the GCPW stub at a variable position, it can be employed as a tunable inductor. The full-wave simulation model also includes the $25 \mu \mathrm{m}$ bonding wires, which connect the photodetector to the PCB, the lightcured epoxy (estimated dielectric constant of 3 ), which sits around the bonding wires and attaches the MMF pigtail to the top-illuminated photodetector, and the transition from the microstrip line to the probe feed of the antenna. Full details of the design and optimization of the matching network are provided in [3]. A photocurrent source $i_{p}$, connected in parallel to the junction capacitance $C_{P}$, serves as the excitation for the DL-RAU, as shown in Fig. 1b. Furthermore, an optical back-to-back connection between the photodetector and a V50-850M VCSEL, biased at $6.0 \mathrm{~mA}$, as employed during the measurements, is included in the model. The VCSEL is described by its small-signal equivalent circuit model. The optical back-to-back connection is modeled by a currentcontrolled current source, which enforces $i_{p}=R \cdot S E \cdot i_{m}$, with $R$ the photodetector's responsivity, $S E$ the VCSEL's slope efficiency, and $i_{m}$ the current which modulates the optical output power of the VCSEL. The full-wave simulation is performed first and results in a five-port scattering parameter description of the distributed parts of the DL-RAU. Subsequently, a small-signal circuit simulation is executed which combines this five-port description with the lumped component models of the VCSEL, optical interconnection, photodetector 


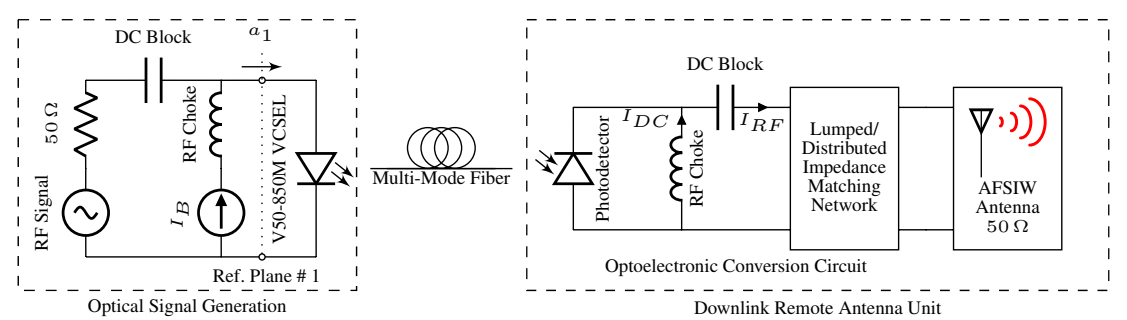

(a)

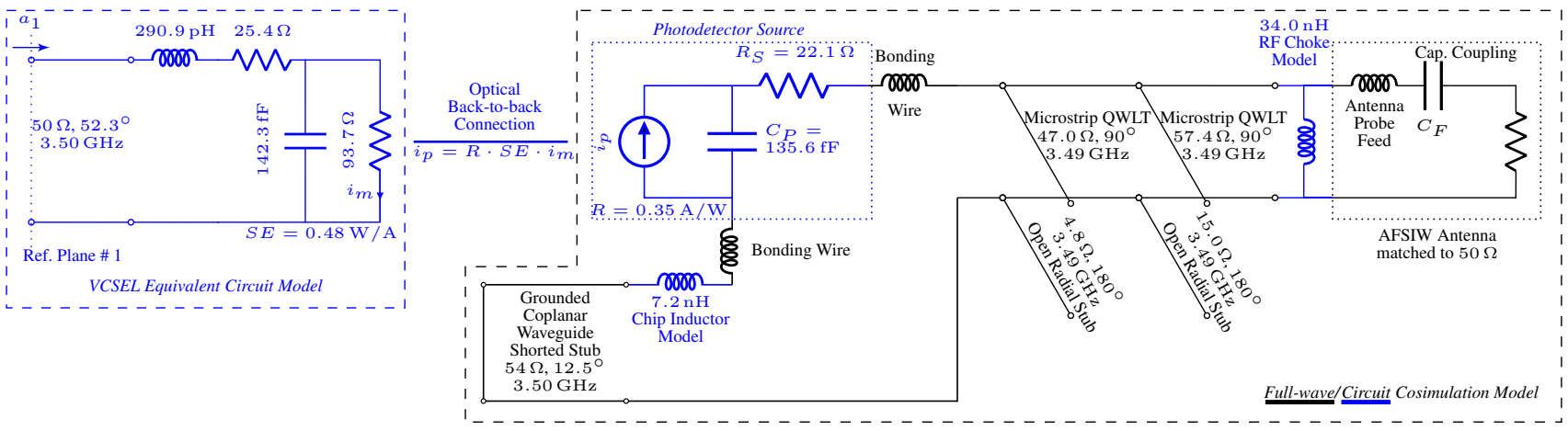

(b)

Fig. 1. (a) High-level schematic overview of the DL-RAU [3]. (b) Full-wave/circuit cosimulation model of the DL-RAU, including the equivalent circuit of the VCSEL (slope efficiency $0.48 \mathrm{~W} / \mathrm{A}$ ), an optical back-to-back connection, and the equivalent circuit of the photodetector (responsivity $0.35 \mathrm{~A} / \mathrm{W}$ ). The components highlighted in black are taken into account by the full-wave simulation, and the parts in blue by the circuit simulation.

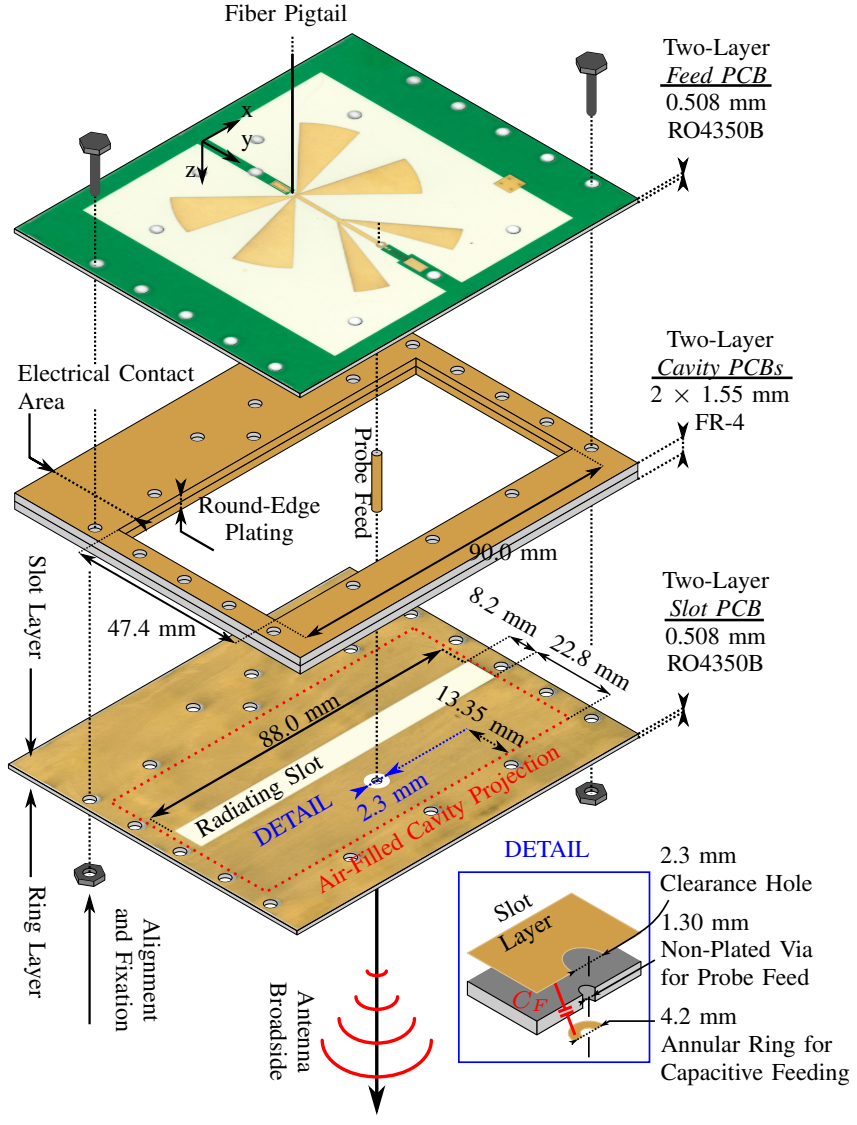

Fig. 2. Structural view of the DL-RAU [3] showing the feed PCB containing the optoelectronic conversion circuit, and the cavity and slot PCBs forming the air-filled substrate-integrated-waveguide cavity-backed slot antenna.

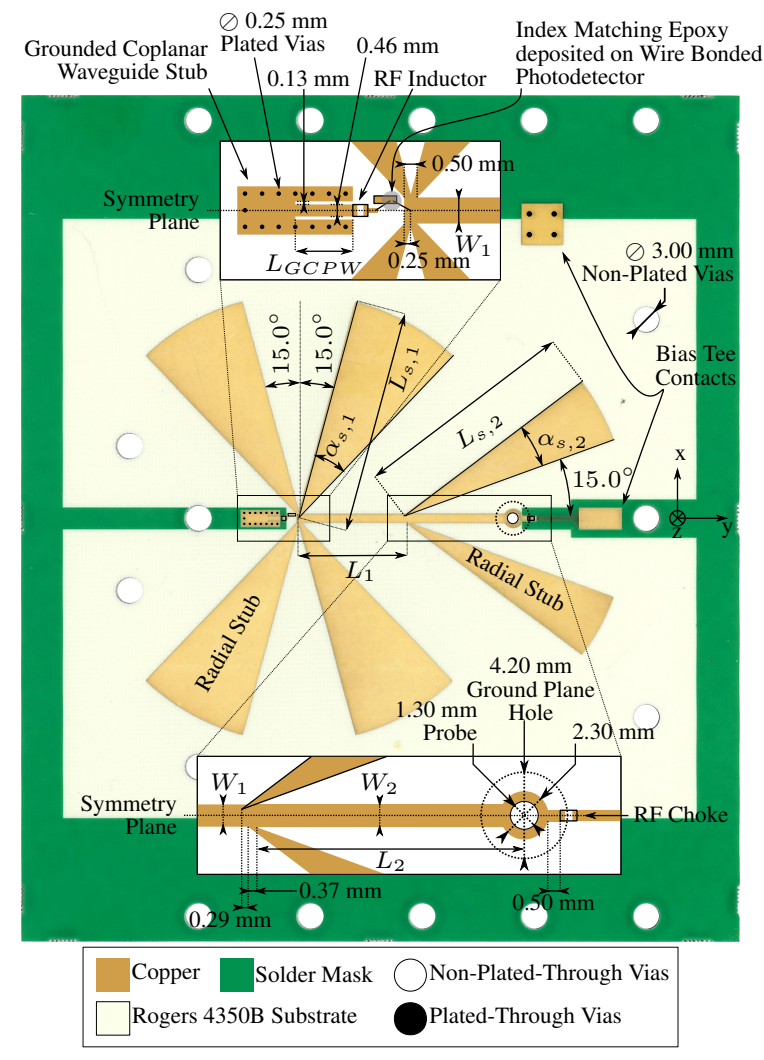

Fig. 3. Detailed view of the feed PCB containing the matching network, bias tee, and photodetector. Optimized dimensions: $L_{G C P W}=2.00 \mathrm{~mm}$, $L_{s, 1}=26.97 \mathrm{~mm}, \alpha_{s, 1}=28.69^{\circ}, L_{1}=13.17 \mathrm{~mm}, W_{1}=1.03 \mathrm{~mm}, L_{s, 2}$ $=26.82 \mathrm{~mm}, \alpha_{s, 2}=17.10^{\circ}, L_{2}=12.50 \mathrm{~mm}$, and $W_{2}=1.07 \mathrm{~mm}$. 
and chip inductors. During a final post-processing step, the DL-RAU's far-field radiation pattern is calculated by applying the excitations at the ports of the full-wave model that were obtained with the circuit simulation. The reference plane of the entire model is located at the VCSEL's modulation input.

\section{Full-wave/circuit Cosimulation Model Results}

The measurements presented in [3] are now compared to the results obtained with the extended simulation model. The agreement of the broadside gain as a function of frequency (Fig. 4a) is slightly reduced within the RF signal frequency band, as compared to [3]. Nevertheless, the gain predicted in [3] employs measurements of the insertion loss of the OECC and the broadside gain of the AFSIW antenna, whereas the result presented here is fully simulated. Several far-field properties at $3.5 \mathrm{GHz}$ are summarized in Table I, for both the standalone AFSIW antenna and the DL-RAU. These include the broadside gain, the front-to-back ratio (FTBR, defined as the ratio of the gain in the $+z$ direction to the gain in the $-z$ direction), the cross-polarization level, and the half-power beam widths (HPBW) in the E-plane ( $Y Z$-plane containing the far-field electric field vector) and the $\mathrm{H}$-plane ( $X Z$-plane containing the far-field magnetic field vector). Finally, the simulated far-field gain patterns in the E- and $\mathrm{H}$-planes are compared to the measurements in Figs. $4 \mathrm{~b}$ and $4 \mathrm{c}$, respectively. As evident in Table I, the extended simulation model partly explains the increased measured HPBW in the E- and H-planes. Still, some deviation remains for the HPBW in the E-plane, and is explained by the measurement fixture, the VCSEL, and the wound-up fiber pigtail that were in close proximity to the RAU during the radiation pattern measurements [3].

TABLE I

MEASURED (SIMULATED) FAR-FIELD PROPERTIES AT $3.50 \mathrm{GHZ}$

\begin{tabular}{r|l|l} 
Characteristic & AFSIW Antenna & DL-RAU \\
\hline Broadside Gain [dBi] & $8.1(8.0)$ & $-0.2(0.0)$ \\
\hline FTBR [dB] & $20.0(15.0)$ & $8.8(17.2)$ \\
\hline Cross-polarization level $[\mathrm{dB}]$ & $-45.4(-63.1)$ & $-28.8(-48.5)$ \\
\hline E/H-plane beam width $\left[^{\circ}\right]$ & $95(85) / 50(50)$ & $150(105) / 55(56)$
\end{tabular}

\section{CONCLUSION}

First, we have outlined the structure and the design of the fully passive DL-RAU proposed in [3]. Furthermore, we have assessed the performance of the DL-RAU with a more extensive full-wave/circuit cosimulation model, which encompasses the VCSEL, optical back-to-back connection, the photodetector, the lumped/distributed matching network, and the AFSIW antenna. In contrast to [3], which interconnected separate models of the AFSIW antenna and the OECC, the presented complete model also includes the spurious radiation by the distributed matching network and the slight frequencydependent input impedance of the antenna, which is not perfectly $50 \Omega$ in the entire frequency band of operation.

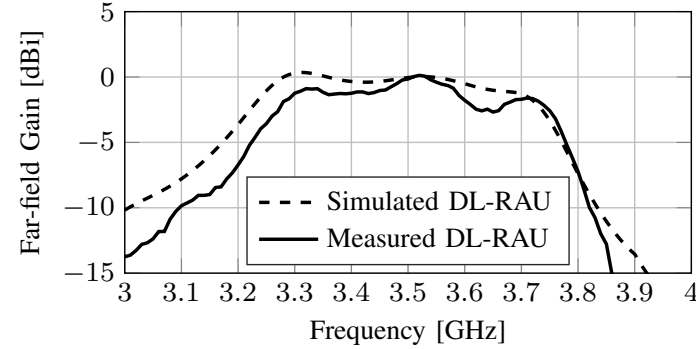

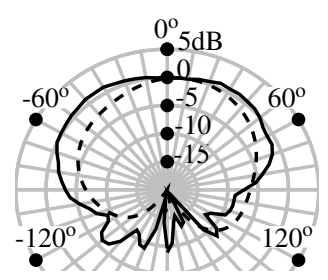

(b) (a)

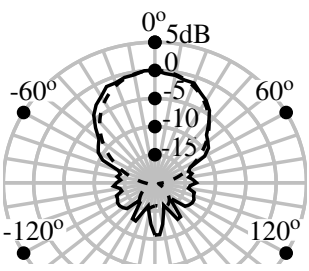

(c)
Fig. 4. Full-wave simulation (- - ) and measurement results (-). (a) Farfield broadside gain [dBi], as a function of frequency. (b) Normalized far-field gain pattern $[\mathrm{dB}]$ at $3.50 \mathrm{GHz}$ in the E-plane, and (c) the H-plane.

\section{ACKNOWLEDGEMENT}

The authors would like to thank the ERC for the advanced grant 695495 "ATTO: A new concept for ultra-high capacity wireless networks".

\section{REFERENCES}

[1] G. Torfs, H. Li, S. Agneessens, J. Bauwelinck, L. Breyne, O. Caytan, W. Joseph, S. Lemey, H. Rogier, A. Thielens, D. Vande Ginste, J. Van Kerrebrouck, G. Vermeeren, X. Yin, and P. Demeester, "ATTO: Wireless networking at fiber speed," J. Lightw. Technol., vol. 36, pp. 1468-1477, April 2018.

[2] C. Lim, A. Nirmalathas, M. Bakaul, P. Gamage, K. L. Lee, Y. Yang, D. Novak, and R. Waterhouse, "Fiber-wireless networks and subsystem technologies," J. Lightw. Technol., vol. 28, pp. 390-405, Feb 2010.

[3] O. Caytan, L. Bogaert, H. Li, J. Van Kerrebrouck, S. Lemey, G. Torfs, J. Bauwelinck, P. Demeester, S. Agneessens, D. Vande Ginste, and H. Rogier, "Passive opto-antenna as downlink remote antenna unit for radio frequency over fiber," J. Lightw. Technol., vol. 36, pp. 4445-4459, Oct 2018.

[4] D. Wake, N. G. Walker, and I. C. Smith, "Zero-bias edge-coupled InGaAs photodiodes in millimetre-wave radio-fibre systems," Electron. Lett., vol. 29, pp. 1879-1881, Oct 1993.

[5] A. Hirata, H. Ishii, and T. Nagatsuma, "Design and characterization of a 120-GHz millimeter-wave antenna for integrated photonic transmitters," IEEE Trans. Microw. Theory Techn., vol. 49, pp. 2157-2162, Nov 2001.

[6] S. Shi, J. Bai, R. Nelson, C. Schuetz, P. Yao, G. J. Schneider, Y. Zhang, and D. W. Prather, "Ultrawideband optically fed tightly coupled phased array," J. Lightw. Technol., vol. 33, pp. 4781-4790, Dec 2015.

[7] S. Shi, J. Bai, G. J. Schneider, Y. Zhang, R. Nelson, J. Wilson, C. Schuetz, D. W. Grund, and D. W. Prather, "Conformal wideband optically addressed transmitting phased array with photonic receiver," $J$. Lightw. Technol., vol. 32, pp. 3468-3477, Oct 2014.

[8] Q. Van den Brande, S. Lemey, J. Vanfleteren, and H. Rogier, "Highly-efficient impulse-radio ultra-wideband cavity-backed slot antenna in stacked air-filled substrate-integrated-waveguide technology," IEEE Trans. Antennas Propag., vol. 66, pp. 2199-2209, May 2018.

[9] A. Belenguer, H. Esteban, and V. E. Boria, "Novel empty substrate integrated waveguide for high-performance microwave integrated circuits," IEEE Trans. Microw. Theory Techn., vol. 62, pp. 832-839, April 2014.

[10] G. A. E. Vandenbosch and A. R. Van de Capelle, "Study of the capacitively fed microstrip antenna element," IEEE Trans. Antennas Propag., vol. 42, pp. 1648-1652, Dec 1994.

[11] G. Matthaei, L. Young, and E. Jones, Microwave filters, impedancematching networks, and coupling structures. Artech House Microwave Library, McGraw-Hill, 1964. 